\title{
Cherenkov luminescence imaging is a fast and relevant preclinical tool to assess tumour hypoxia in vivo
}

\author{
Emiko Desvaux ${ }^{1 \dagger}$, Alan Courteau ${ }^{1 \dagger}$, Pierre-Simon Bellaye ${ }^{1}$, Mélanie Guillemin ${ }^{1}$, Camille Drouet ${ }^{1}$, Paul Walker ${ }^{2,3}$, \\ Bertrand Collin ${ }^{1,4}$ and Richard A. Decréau ${ }^{4^{*}}$
}

\begin{abstract}
Purpose: Molecular imaging techniques visualise biomarkers for both drug development and personalised medicine. In this field, Cherenkov luminescence imaging (CLI) seems to be very attractive by allowing imaging with clinical PET radiotracers with high-throughput capabilities. In this context, we developed a fast CLI method to detect tumour hypoxia with ${ }^{18}$ F-fluoromisonidazole (FMISO) for drug development purposes.

Methods: Colon cancer model was induced in mice by subcutaneous injection of $1 \times 10^{6} \mathrm{CT}-26$ cells. FMISO was injected, and simultaneous PET-blood oxygen level dependent (BOLD)-MRI followed by CLI were performed along with immunohistochemistry staining with pimonidazole.

Results: There was a significant correlation between FMISO PET and CLI tumour uptakes, consistent with the BOLD-MRI mapping. Tumour-to-background ratio was significantly higher for CLI compared with PET and MRI. Immunohistochemistry confirmed tumour hypoxia. The imaging workflow with CLI was about eight times faster than the PET-MRI procedure.
\end{abstract}

Conclusion: $\mathrm{CLI}$ is a fast and relevant tool to assess tumour hypoxia. This approach could be particularly interesting for hypoxia-targeting drug development.

Keywords: Cherenkov luminescence imaging (CLI), ${ }^{18} \mathrm{~F}$-Fluoromisonidazole (FMISO), Hypoxia, Positron emission tomography (PET), Magnetic resonance imaging, BOLD, Colon cancer

\section{Introduction}

Molecular imaging contributes to develop safer and effective drugs while shortening the time-to-market [1]. Cherenkov luminescence imaging (CLI) has emerged as a promising optical imaging (OI) modality. CLI is based on the intrinsic capability of radionuclides (e.g. fluorine 18) to emit light through the Cherenkov effect [2]. CLI shares the advantages of OI with high sensitivity, low cost and high throughput [2]. CLI is widely used in preclinical oncology, although it has never been used to investigate tumour hypoxia. Hypoxia is encountered in aggressive tumours and is responsible for the resistance

\footnotetext{
* Correspondence: Richard.Decreau@u-bourgogne.fr

${ }^{\dagger}$ Emiko Desvaux and Alan Courteau contributed equally to this work.

${ }^{4}$ Institut de Chimie Moléculaire de l'Université de Bourgogne (ICMUB), 9

Avenue Alain Savary, 21078 Dijon, France

Full list of author information is available at the end of the article
}

to treatments [3]. The tumour hypoxic status can be determined with ${ }^{18} \mathrm{~F}$-fluoromisonidazole (FMISO) positron emission tomography (PET) and/or blood oxygen level dependent (BOLD) magnetic resonance imaging (MRI) [3]. Albeit appealing in several regards, such techniques suffer from limited throughputs [4]. Indeed, PET/MRI imaging requires expensive systems and is usually characterised by measurement times ranging from 20 to 30 min for static imaging studies and up to $60-90 \mathrm{~min}$ when involving dynamic PET assessments or/and functional MRI imaging [4]. Thus, CLI could represent a fast, cost-effective and decisive tool to predict the efficacy of hypoxia-activated anticancer drugs since baseline hypoxia imaging is of crucial interest to successfully develop hypoxia-targeted drugs in both preclinical [5] and clinical settings [6]. In addition, the use of CLI in humans has also recently been demonstrated, providing CLI with 
an important translational aspect [7]. Herein, we present the first report of preclinical hypoxia imaging by CLI and its cross-validation by simultaneous PET-MRI imaging following injection of FMISO.

\section{Materials and methods}

\section{Animal experiments}

Animal experiments were approved by our institution (Centre Georges-François Leclerc, Dijon, France) and complied with the Ethical Committee and the French Ministry of Higher Education and Research. Six- to 7 -week-old-BALB/c female mice $(n=13)$ were obtained from the animal husbandry of the University of Burgundy (Dijon, France). A total of $1 \times 10^{6}$ murine CT26 (ATCC, CRL-2638, USA) colon cancer cells were implanted subcutaneously at the right flank of depilated mice. The tumour grew over 18 days until it reached a mean volume of $316 \pm 81 \mathrm{~mm}^{3}$. Then, mice were injected intravenously under anaesthesia (2\% isoflurane in air) with $10 \mathrm{MBq}$ of FMISO and pimonidazole $(60 \mathrm{mg} / \mathrm{kg})$. PET-MRI imaging was performed 120 min post-injection (p.i.), and CLI imaging was performed on the same animal right after the PET-MRI exam (160 min p.i.). The tumour area was depilated $24 \mathrm{~h}$ before CLI imaging. All images were decay corrected for quantification. After the imaging sessions, mice were sacrificed and tumours were formalin-fixed $(48 \mathrm{~h})$ for pimonidazole immunohistochemistry. ${ }^{18}$ F-Fluoromisonidazole (FMISO) was synthesised by Pharmimage ${ }^{\odot}$ within its cyclotron and radiopharmacy platform following an in-house synthetic scheme (Additional file 1).

\section{PET-MRI imaging}

PET and MRI images were simultaneously acquired in a dual-ring SiPM microPET fully integrated in a $7 \mathrm{~T}$ preclinical MRI (MR Solutions, Guildford, UK). During imaging, mice were anaesthetised with $2 \%$ isoflurane in air. In addition to $\mathrm{T} 1$ anatomical images, BOLD-MRI sequences have been performed using multi-gradient echo (MGE) sequence (T2\%-weighted acquisition) with the following parameters: $\mathrm{TR}=500 \mathrm{~ms}$; $\mathrm{TE}=3,6,9,12,15$ and $18 \mathrm{~ms}$; flip angle $=40^{\circ}$; and image matrix $=128 \times 128$. MRI voxel values have been interpolated using a Python 3 homemade script, to obtain a T2* map (BOLD data). PET images have been reconstructed using a 3D OSEM algorithm (2 iterations, 32 subsets). PET quantifications were performed using Vivoquant ${ }^{\mathrm{Ts}}$ software (Invicro, Boston, MA, USA), and results have been expressed in $\%$ injected activity of FMISO per $\mathrm{mm}^{3}\left(\% \mathrm{IA} / \mathrm{mm}^{3}\right)$.

\section{CLI imaging}

Following PET-MRI imaging, mice underwent CLI imaging performed with an optical imager (IVIS Lumina $\mathrm{III}^{\circ}$, PerkinElmer, USA) equipped with a CCD camera with the following parameters: exposure time $300 \mathrm{~s}$, binning factor 16 , field of view $7.5 \mathrm{~cm}$, in open filter mode. Mice were maintained under anaesthesia throughout the procedure (2\% isoflurane in air). Results are expressed in radiance $\left(\mathrm{p} / \mathrm{s} / \mathrm{cm}^{2} / \mathrm{sr}\right)$. Signal-to-noise ratio for PET, MRI and CLI following the injection of FMISO was determined by the ratio of the signal from the tumour and a contralateral irrelevant region of interest (muscle).

\section{Pimonidazole immunostaining}

Tumour sections from mice injected with pimonidazole (given at $60 \mathrm{mg} / \mathrm{kg}$ i.p., $120 \mathrm{~min}$ before sacrifice) were deparaffinised and immunostained with a commercial kit (Hydoxyprobe plus kit, USA) to specifically stain pimonidazole and determine the level of hypoxia. Stained sections were analysed using Axioscope $\mathrm{A} 1^{\circ}$ coupled to an Axiocam $503^{\circ}$ colour camera and $\mathrm{ZEN}^{\circ}$ Software (Zeiss, Germany).

\section{Statistical analysis}

For all the experiments performed within this study, statistical analyses were achieved using GraphPad Prism ${ }^{\circ}$ 7.0 (GraphPad Software, USA) and a $p$ value of less than 0.05 was considered significant. Comparisons between more than two groups have been performed using one-way ANOVA, and correlations were determined by the Pearson's coefficient using GraphPad Prism ${ }^{\oplus}$ 7.0.

\section{Results}

$\mathrm{CLI}$ imaging of tumour hypoxia and cross-validation with simultaneous PET-MRI

The tumour uptake of FMISO was measured with both PET and CLI. Through our work, we managed to highlight a positive correlation $(n=13$; Pearson's coefficient $=0.8533, p<0.0001$ ) between FMISO uptake obtained with PET and CLI (Fig. 1). No matter what imaging modality was used, all tumours exhibited hypoxia in our model as shown by both FMISO tumour uptake measured by PET (Fig. 2a, b) and BOLD MRI mapping (Fig. 2c, d) or CLI (Fig. 2e). The BOLD signal obtained from $\mathrm{T} 2 *$-weighted images reflects the heterogeneity in the magnetic field $B_{0}$ caused by changes in the level of $\mathrm{O}_{2}$ in the blood. The signal decrease on $\mathrm{T} 2 *$ maps hence reflects deoxyhaemoglobin paramagnetic characteristics, thus providing indirect information about haemoglobin saturation rate, reflective of tumour oxygenation. Interestingly, we demonstrated that hypoxic areas within tumours measured with FMISO-PET corresponded with the area of decreased BOLD signal obtained with the MRI (Fig. 2b, d, black circle). Nevertheless, some FMISO-PET positive areas showed corresponding increased BOLD signal reflecting properly oxygenated areas thus highlighting discrepancies between cellular hypoxia measured by FMISO-PET and 


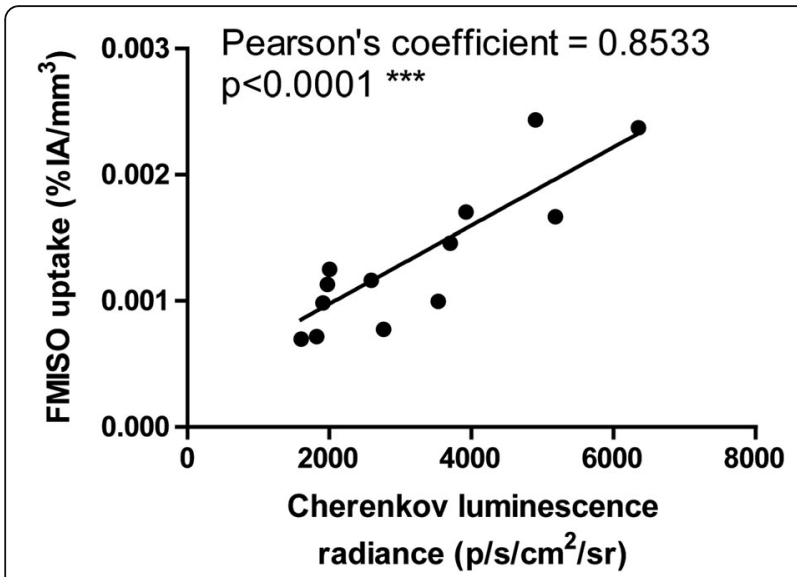

Fig. 1 Correlation between Cherenkov luminescence imaging and PET following the injection of FMISO ( $n=13$, Pearson's coefficient $\left.=0.8533, p<0.0001^{* * *}\right)$. Results are presented as radiance $\left(\mathrm{p} / \mathrm{s} / \mathrm{cm}^{2} / \mathrm{sr}\right)$ for $\mathrm{CLI}$ and $\% \mathrm{IA} / \mathrm{mm}^{3}$ for FMISO-PET

blood oxygen levels measured by MRI (Fig. 2b, d, white arrow). Most importantly, while the overall PET-MRI procedure took approximately $40 \mathrm{~min}$ per animal $(39.85 \pm 1.81 \mathrm{~min})$, the CLI procedure only lasted for 5 min per animal. Tumour-to-background ratio (TBR) has been measured for all imaging modalities, and most interestingly, CLI displayed a significantly higher TBR demonstrating the sensitivity of this imaging technique (Fig. 2f).

\section{Hypoxia immunostaining with pimonidazole}

The ex vivo study performed on slices of CT26 colon carcinoma further confirmed CLI-PET-MRI findings showing diffused hypoxic areas heterogeneously distributed within the tissue (Fig. 3) with either fluorescence microscopy (Fig. 3a) or transmitted light microscopy (Fig. 3b). Interestingly, we demonstrated a moderate correlation ( $n=9$; Pearson's coefficient $=0.7238, p<0.05)$ between pimonidazole fluorescence microscopy and FMISO-CLI (Fig. 3c).

\section{Discussion}

Biomarkers of tumour hypoxia allow to improve the drug development process through the selection of individuals potentially eligible for therapies that target hypoxic environment in both preclinical and clinical settings [5]. FMISO is a well-known PET radiotracer of tumour hypoxia [3] able to produce a CR due to the emission of a positron with a maximal energy of $633 \mathrm{keV}$ [2]. The present study is the first report of CLI showing the FMISO uptake in hypoxic tumours, cross-validated by simultaneous PET-T2* MRI, further confirmed by classical pimonidazole immunostaining [3]. We assume that the advantages of CLI are twofold: (i) fast imaging since the acquisition time for simultaneous PET-MRI imaging was about $40 \mathrm{~min}$, whereas that of CLI was as low as 5 min and (ii) CLI signal remains proportional to the PET signal since both significantly correlated, consistent with previous data $[2,8]$. Our results are in accordance with other studies, which demonstrated a clear linear correlation between CLI radiance and PET using several radionuclides including 18-fluorine, 64-copper or 89-zirconium in vivo [9-11]. A close correlation between CLI and $\gamma$-counter-based biodistribution analysis has also been highlighted by several studies further validating this fast and cheap technique as a reliable alternative for high-throughput analysis $[9,11]$. However, CLI imaging suffers from limitations. Compared with PET/ MRI, which provides a 3D image, CLI only gives a $2 \mathrm{D}$ image hampering accurate quantification of the signal. In addition, CLI-based imaging relies on limited tissue penetration and low sensitivity for deep targets as well as the high amount of diffusely scattered photons in the mouse tissues. Therefore, FMISO-CLI only gives a qualitative measure while FMISO-PET gives a fully 3D quantitative measurement of tumour hypoxia. Nevertheless, our study demonstrates that CLI provides favourable tumour-to-background ratio for shallow signals (e.g. subcutaneous tumours), compared with that of PET and MRI. As a consequence, FMISO-CLI appears as a sensitive and powerful tool to rapidly and cost-effectively discriminate subcutaneous tumours with hypoxia from tumours without hypoxia in the context of hypoxia-targeting drug discovery. Importantly, the improved workflow brought by CLI not only provides faster preclinical screening of hypoxia, but also considerably reduces the time each animal remains under anaesthesia, which is a critical parameter when evaluating tumour hypoxic status. As a consequence, CLI may provide more reliable data regarding baseline hypoxia, which in turn may improve hypoxia-related drug development. Given the relative heterogeneity of hypoxic signals among animals/patients in tumours in both clinical and preclinical settings, it appears crucial that the development of hypoxia-targeting drugs relies on fast and relevant biomarkers of hypoxia. Such a strategy involving FMISO-CLI would allow a rapid selection of subjects with positive hypoxic signal in order to successfully evaluate hypoxia-targeting drugs. This was recently highlighted by Grkovski et al. who suggested that the poor outcome of the MAESTRO trial, evaluating the hypoxia-activated evofosfamide, was partly due to the inclusion of non-hypoxic tumours, which may have masked the benefit of the drug [5]. Such a result shows that not only CLI is relevant to assess tumour hypoxia, but also for preclinical drug development due to its high throughput capabilities also reported elsewhere [8]. Strategies are underway to make CLI an even more potent technique for oncologic studies [12]. 


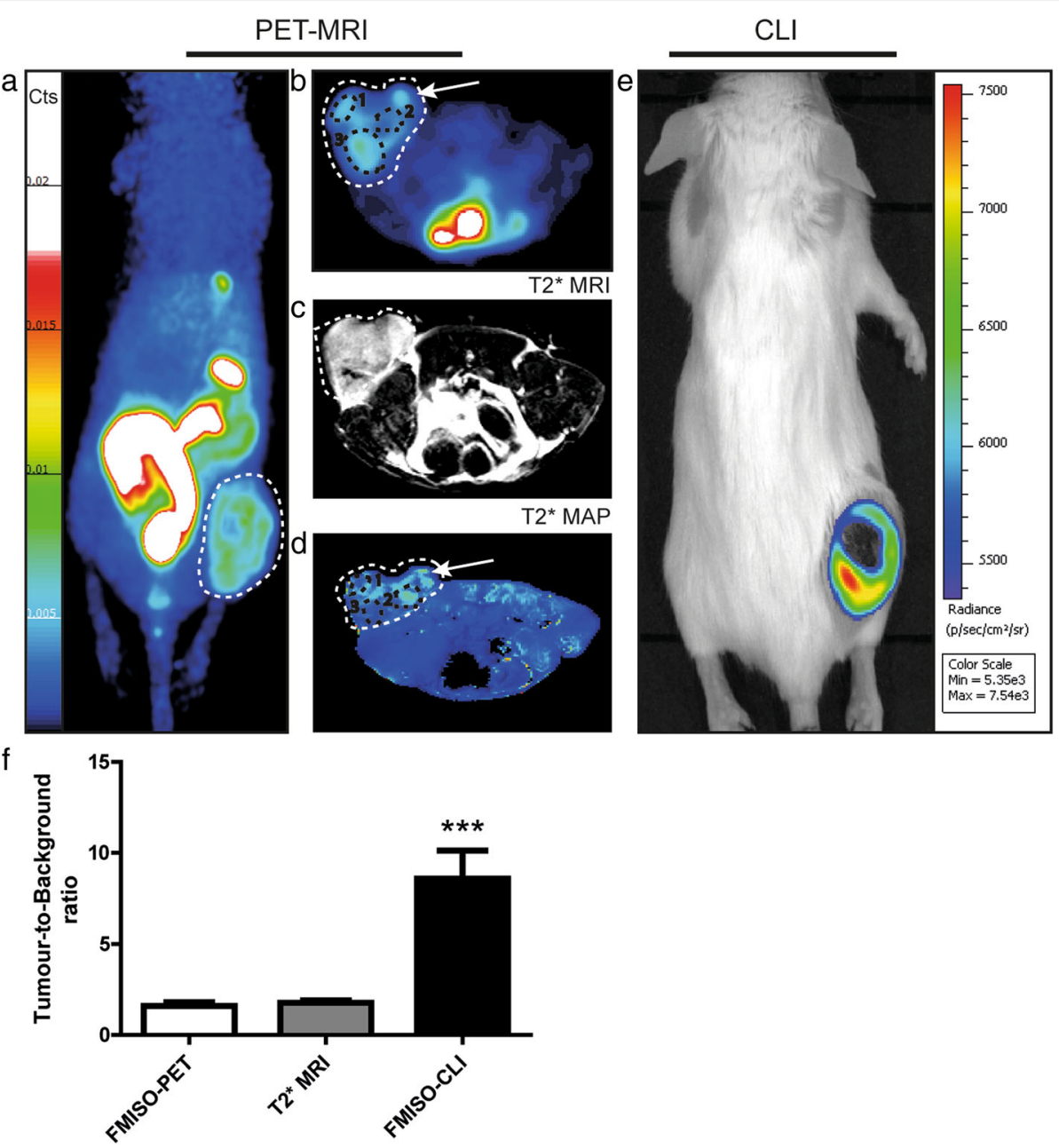

Fig. 2 Imaging of hypoxia within CT26 tumour bearing mice $(n=13)$, PET-MRI a-d Representative PET-MRI images showing the global co-localisation of FMISO uptake and BOLD MRI signal. Images were acquired 120 min post-injection of $10 \mathrm{MBq}$ of $\left[{ }^{18} \mathrm{~F}\right]$-Fluoromisonidazole (FMISO). PET. a Representative maximum-intensity-projection FMISO PET Image; b Transversal slice showing FMISO uptake within the tumour; $\mathbf{c}$ T2*weighted MRI and $\mathbf{d}$ BOLD image derived from T2* mapping. White dashed lines: tumour limits, white arrows: oxygenated tumour area (increased BOLD signal), black circle: hypoxic tumour areas (decreased BOLD signal). CLI (e): Representative FMISO CLI image of the same mouse as for a to $\mathbf{d}$ acquired just after the PET-MRI scan. $\mathbf{f}$ Tumour-to-background ratio for PET, MRI and CLI following the injection of FMISO determined by the ratio of the signal from the tumour and a contralateral irrelevant region of interest (muscle); $n=13,{ }^{* * *} p<0.001$

An innovative aspect of our study relies on the application of CLI to tumour hypoxia and the cross-validation with classical pimonidazole immunostaining. As mentioned previously, correlation between CLI and PET is already documented but, to our knowledge, the current study is the first to validate CLI as an accurate tool to quickly detect tumour hypoxia. The correlation between CLI and pimonidazole immunostaining that we demonstrate, while significant, is weaker than that between CLI and PET. The major issue with classical immunostaining quantification, widely used in clinic, relies on the fact that it is only representative of a small portion of the tissue potentially introducing bias in analysis. Tumour hypoxia is heterogeneously distributed within tumours and is often subjected to rapid changes depending on various environmental parameters (e.g. temperature, activity status of the animal). Therefore, rapid and non-invasive evaluation of tumour hypoxia is crucial to obtain the most accurate signal. Histological measurement of hypoxia requires to euthanize animals, resect the tumour and then fix the tissue in formalin-based solution. Even though these procedures may only require a short period of time, the hypoxic status of the tumour may suffer from some changes (e.g. short lack of oxygen following animal euthanasia). Therefore, we believe that CLI imaging of FMISO is a more representative measure of in vivo tumour hypoxia compared with histology. 

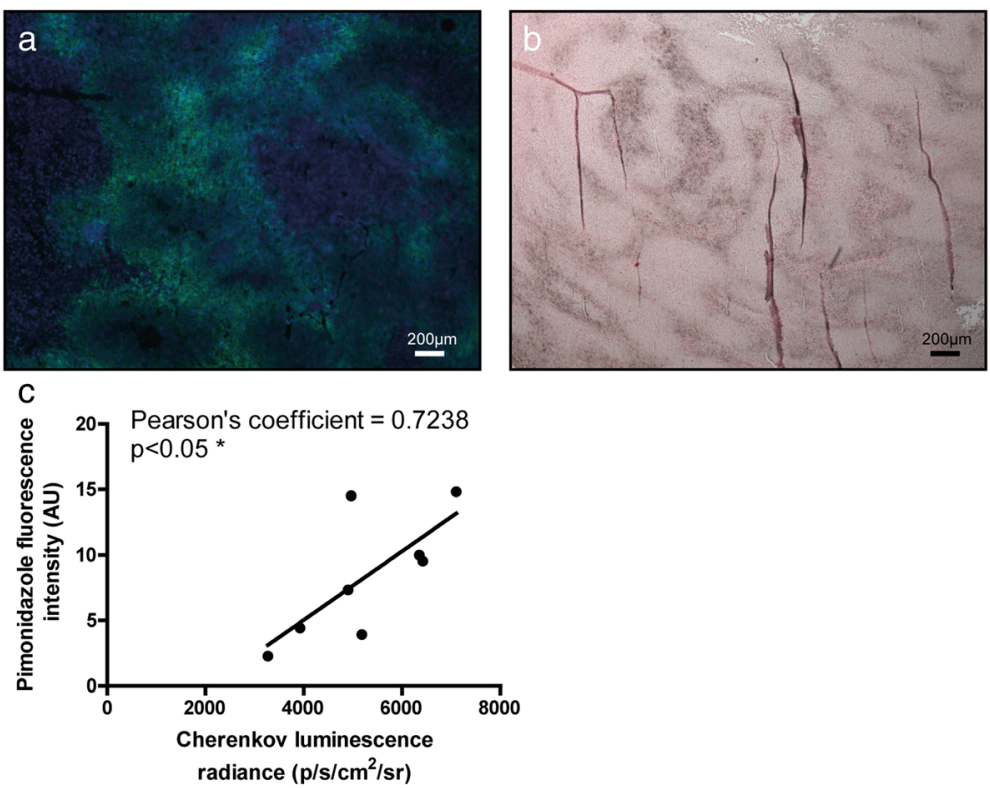

Fig. 3 Microscopic slides of tumours for the same mouse as for Fig. 2a, b with pimonidazole staining ( $\times 50$ magnification). Hypoxic regions are shown either in green (a fluorescence microscopy) or in brown (b transmitted light microscopy). c Correlation between Cherenkov luminescence imaging following the injection of FMISO and fluorescence pimonidazole staining $\left(n=9\right.$, Pearson's coefficient $\left.=0.7238, p<0.05^{*}\right)$. Results are presented as radiance $\left(\mathrm{p} / \mathrm{s} / \mathrm{cm}^{2} / \mathrm{sr}\right.$ ) for $\mathrm{CLI}$ and fluorescence intensity (arbitrary units, $\mathrm{AU}$ ) of pimonidazole

Important questions can be raised about the clinical applicability of CLI in humans given the differences between preclinical and clinical studies regarding, for instance, injected doses and depth of the sites of interest. In our preclinical study, CLI imaging was performed on a subcutaneous tumour after injection of $10 \mathrm{MBq}$ of FMISO $(400 \mathrm{MBq} / \mathrm{kg}$ in a mouse of 25 g). As a comparison, current guidelines prescribe a dose of $3.7 \mathrm{MBq} / \mathrm{kg}$ in patients for standard PET exams. With a 100-fold lower injected activity and deeper tumour site, CLI imaging in humans remains a challenge and often requires the use of ultrasensitive photon-detecting devices and strict restriction of ambient light [7]. Nevertheless, the proof-of-concept for the clinical use of CLI has already been described especially in the field of guided surgery [13] where tumour hypoxia might be a relevant target [14]. The use of CLI in the context of guided surgery may indeed represent a way to circumvent the limited tissue penetration and tissue diffusion of CR. Based on our results, FMISO-CLI could therefore be a fast, cheap and sensitive method to specifically and accurately remove otherwise undetectable hypoxic malignant lesions. In addition, with the recent rise of intraoperative radiation therapy, the detection of radio-resistant hypoxic tumour areas by imaging such as FMISO-CLI might be of great interest in order to provide radiation boosts which would improve radiation therapy outcome [15].

\section{Conclusion}

Tumour hypoxia can be assessed with CLI induced by FMISO since it is cross-validated by PET-MRI and histology. Interestingly, the workflow for CLI imaging is much more efficient than PET-MRI paving the way for this method to speed-up and strengthen the preclinical development of hypoxia-targeting drugs.

\section{Additional file}

Additional file 1: Synthesis and purification of ${ }^{18} \mathrm{~F}$-Fluoromisonidazole (FMISO). (DOCX $344 \mathrm{~kb})$

\section{Acknowledgements}

Not applicable.

\section{Funding}

This work was supported by the Burgundy General Council CRB (funding), Cyclopharma ${ }^{\oplus}$ (CO-funding, FMISO) and CNRS (Chaire d'Excellence Program: RD). The 3MIM (CNRS, UB, CRB) and PARI2 programmes, and the Pharm/mage consortium are acknowledged. This work was also supported by the European Union through the PO FEDER-FSE Bourgogne 2014/2020 programme, and by a French Government grant managed by the French National Research Agency (ANR) under the programme 'Investissements d'Avenir' (with reference ANR-10-EQPX-05-01/IMAPPI Equipex) and by the Fondation de Coopération Scientifique Bourgogne Franche-Comté.

Availability of data and materials

Please contact author for data request.

Authors' contributions

$E D, P S B, M G, A C$ and $C D$ performed the experiments. PSB, BC and RD wrote the paper. ED, PSB, BC, RD, AC and PW conceived either all or parts of the study. All authors read, contributed to, and approved the final manuscript. 


\section{Ethics approval}

Name of the institutions or licensing bodies that approved the experimental protocols:

Nuclear safety (a) Safety rules and protocols were set at and approved by the Centre George-François Leclerc (CGFL) and applied at CGFL Preclinical Imaging Platform; (b) The protocols strictly comply with the regulations governing radiopharmaceuticals and standards of the French Nuclear Safety Agency (ASN) that approves CGFL protocols.

In vivo studies All animal studies were conducted at and approved by the Centre George François Leclerc (CGFL) in accordance with the relevant guidelines and legislation on the use of laboratory animals (directive 2010/ 63/EU) and were approved by accredited the Ethical Committee (C2ea Grand Campus $\left.\mathrm{n}^{\circ} 105\right)$ and French Ministry of Higher Education and Research.

\section{Consent for publication}

Not applicable.

\section{Competing interests}

The authors declare that they have no competing interest.

\section{Publisher's Note}

Springer Nature remains neutral with regard to jurisdictional claims in published maps and institutional affiliations.

\section{Author details}

'Centre George François Leclerc (CGFL), 1 rue du Professeur Marion, 21079 Dijon, France. ${ }^{2}$ Université Bourgogne Franche Comté, CNRS, Laboratoire Electronique Informatique \& Image (Le2i), UMR, 6306 Dijon, France. ${ }^{3}$ Université Hospital Francois Mitterrand, Dijon, France. ${ }^{4}$ Institut de Chimie Moléculaire de I'Université de Bourgogne (ICMUB), 9 Avenue Alain Savary, 21078 Dijon, France.

Received: 4 October 2018 Accepted: 3 December 2018

Published online: 20 December 2018

\section{References}

1. Cunha L, Szigeti K, Mathe D, Metello LF. The role of molecular imaging in modern drug development. Drug Discov Today. 2014;19:936-48. https://doi. org/10.1016/j.drudis.2014.01.003.

2. Ciarrocchi E, Belcari N. Cerenkov luminescence imaging: physics principles and potential applications in biomedical sciences. EJNMMI Phys. 2017;4:14. https://doi.org/10.1186/s40658-017-0181-8.

3. Fleming IN, Manavaki R, Blower PJ, West C, Williams KJ, Harris AL, et al. Imaging tumour hypoxia with positron emission tomography. $\mathrm{Br} J$ Cancer. 2015;112:238-50. https://doi.org/10.1038/bjc.2014.610.

4. Judenhofer MS, Cherry SR. Applications for preclinical PET/MRI. Semin Nucl Med. 2013:43:19-29. https://doi.org/10.1053/.semnuclmed.2012.08.004.

5. Grkovski M, Fanchon L, Pillarsetty NVK, Russell J, Humm JL. (18)Ffluoromisonidazole predicts evofosfamide uptake in pancreatic tumor model. EJNMMI Res. 2018;8:53. https://doi.org/10.1186/s13550-018-0409-1.

6. Van Cutsem E, Verheul HM, Flamen P, Rougier P, Beets-Tan R, Glynne-Jones $R$, et al. Imaging in colorectal cancer: progress and challenges for the clinicians. Cancers. 2016;8. https://doi.org/10.3390/cancers8090081.

7. Thorek DL, Riedl CC, Grimm J. Clinical Cerenkov luminescence imaging of (18)F-FDG. J Nucl Med. 2014;55:95-8. https://doi.org/10.2967/jnumed.113. 127266

8. $\mathrm{X} U \mathrm{Y}, \mathrm{Liu} \mathrm{H}, \mathrm{Cheng} Z$. Harnessing the power of radionuclides for optical imaging: Cerenkov luminescence imaging. J Nucl Med. 2011;52:2009-18, https://doi.org/10.2967/jnumed.111.092965.

9. Maier FC, Schmitt J, Maurer A, Ehrlichmann W, Reischl G, Nikolaou K, et al. Correlation between positron emission tomography and Cerenkov luminescence imaging in vivo and ex vivo using 64Cu-labeled antibodies in a neuroblastoma mouse model. Oncotarget. 2016;7:67403-11. https://doi. org/10.18632/oncotarget.11795.

10. Zhang X, Kuo C, Moore A, Ran C. In vivo optical imaging of interscapular brown adipose tissue with (18)F-FDG via Cerenkov luminescence imaging. PLoS One. 2013;8:e62007. https://doi.org/10.1371/journal.pone.0062007.

11. Natarajan A, Habte F, Liu H, Sathirachinda A, Hu X, Cheng Z, et al. Evaluation of 89Zr-rituximab tracer by Cerenkov luminescence imaging and correlation with PET in a humanized transgenic mouse model to image
NHL. Mol Imaging Biol. 2013;15:468-75. https://doi.org/10.1007/s11307-0130624-0.

12. Bernhard Y, Collin B, Decreau RA. Redshifted Cherenkov radiation for in vivo imaging: coupling Cherenkov radiation energy transfer to multiple Forster resonance energy transfers. Sci Rep. 2017;7:45063. https://doi.org/10.1038/ srep45063.

13. Grootendorst MR, Cariati M, Kothari A, Tuch DS, Purushotham A. Cerenkov luminescence imaging (CLI) for image-guided cancer surgery. Clin Transl Imaging. 2016;4:353-66. https://doi.org/10.1007/s40336-016-0183-X.

14. Lv PC, Roy J, Putt KS, Low PS. Evaluation of a carbonic anhydrase IXtargeted near-infrared dye for fluorescence-guided surgery of hypoxic tumors. Mol Pharm. 2016;13:1618-25. https://doi.org/10.1021/acs. molpharmaceut.6b00065.

15. Chua BH, Kron T. Intraoperative radiotherapy with image guidance: mix and match. J Med Imaging Radiat Oncol. 2018. https://doi.org/10.1111/17549485.12836.

\section{Submit your manuscript to a SpringerOpen ${ }^{\circ}$ journal and benefit from:}

- Convenient online submission

- Rigorous peer review

- Open access: articles freely available online

- High visibility within the field

- Retaining the copyright to your article

Submit your next manuscript at $\boldsymbol{\nabla}$ springeropen.com 